\title{
The CanMED's wisdom of Cool Hand Luke
}

\author{
Keith Rourke, MD, FRCSC \\ Division of Urology, University of Alberta, Edmonton, AB
}

See related article on page 437.

Cite as: Can Urol Assoc J 2013;7(11-12):442-3. htrp://dx.doi.org/10.5489/cuaj.1777

Published online December 5, 2013.

"W hat we've got here is failure to communicate." These words first uttered in the classic 1967 movie Cool Hand Luke are now firmly entrenched in popular culture. They also are relevant to the current training and practice of urologists. Humans have a great ability to master and create language, but sometimes during the most critical times our communication falls short. Lack of communication is the most common reason for medical malpractice claims in Canada and the United States. In fact $42 \%$ of malpractice claims are related to perceived poor communication and disproportionately represent $79 \%$ of malpractice costs. ${ }^{1}$

Roberts and colleagues survey the attitudes, beliefs and experiences of graduating urology residents with reference to the CanMED's Communicator role. ${ }^{2}$ This well-conceptualized study is of interest to any urologist involved in the education of residents. The article describes the large discrepancy between the perceived importance of teaching CanMED's roles and their practical application. Many urologists feel that the Communicator role is the most important of the roles. Based on this article, it appears our residents also hold these same views; $83 \%$ and $90 \%$ of residents, respectively, feel being a good communicator is important to both training and future practice.

It also seems that the rest of Canada agrees. For example, a search of the Canadian Medical Protective Association (CMPA) website using the term "communication" produced 1070 hits. Additionally both the Royal College of Physicians and Surgeons of Canada (RCPSC) and the CMPA have online curricula dedicated to enhancing physician communication skills. ${ }^{3,4}$ Only $15 \%$ of our graduating residents are aware of these resources. How is this possible? Perhaps as a specialty we have not clearly outlined our expectations for the Communicator role. Yet, there are over 20 objectives specifically addressing this role in the current Objectives of Training in Urology. The importance of communication in urology has been clearly defined and conveyed throughout the country.

Despite the obvious importance of communication and widely available educational resources, there is a stark contrast in what is currently taught in residency. Only $31 \%$ of residents perceived that formal training in communication was available to them and only $38 \%$ felt communication was formally addressed during residency. Perhaps the resident preparing to write a qualifying examination has their attention deflected temporarily from non-medical expert content. It may also be that residents simply forgot that they received formal CanMED's teaching.

There is however a lack of evaluation tools used to assess communication during residency training in urology. Urology residency programs use formal tools to teach the Communicator role only $10 \%$ to $31 \%$ of the time. Then again this lack of formal teaching could also be a classic example of the "tail wagging the dog" in medical education. Learners study and prioritize what they are examined on. Unless we evaluate and examine these roles in a more formal and robust manner, these roles may never be deemed important enough to claim the attention they deserve.

At present the main focus of most Canadian qualifying exams is on the Medical Expert role. Unless our residents are examined on the CanMED's roles in a high stakes format, residents may never prioritize these roles. It seems that the Medical Council of Canada has taken notice and is planning to restructure the Canadian qualifying exams to better evaluate physician's communication skills and professionalism. ${ }^{5}$ Can this change in evaluation lead to a new age in medical education? If not, resident education may have to be completely reimagined. 
Competing interests: Dr. Rourke declares no competing financial or personal interests.

\section{References}

1. Hoffman, Jock, Communication Factors in Malpractice Cases, Controlled Risk Insurance Company (CRICO), March 15, 2012. https://www.rmf.harvard.edu/Clinician-Resources/Topic-Tag/Communication. Accessed November 12, 2013.

2. Roberts $G$, Beiko D, Touma N, et al. Are we getting through? A national survey on the CanMEDS communicator role in urology residency. Can Urol Assoc J 2013;7:437-41. http://dx.doi.org/10.5489/cuaj.264
3. Royal College of Physician and Surgeons of Canada, CanMEDS and Faculty Development Resources; 2013. http://www.royalcollege.ca/portal/page/portal/rc/canmeds/resources. Accessed November 13, 2013.

4. CMPA Good Practices Guide; 2012, Ottawa, Canada. http://www.cmpa-acpm.ca/cmpapd04/docs/ ela/goodpracticesguide/pages/communication/communication-e.html. Accessed November 13, 2013.

5. Cross C. Changes ahead for Medical Council of Canada qualifying exams. CMAJ October 25, 2013. http://www.cmai.ca/site/earlyreleases/25octl3_changes-ahead-for-Medical-Council-of-Canadaqualifying-exams.xhtml. Accessed November 13, 2013. http://dx.doi.org/10.1503/cmaj.109-4642

Correspondence: Dr. Keith Francis Rourke, Hys Medical Centre, Suite 400 - 11010 101st Street NW, Edmonton, AB; krourke@ualberta.ca

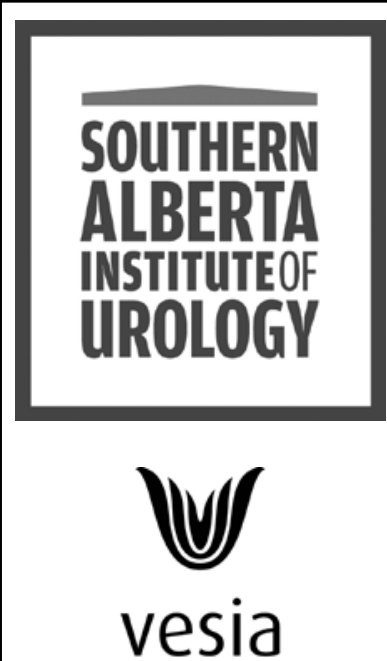

[ ALBERTA BLADDER CENTRE

FACULTY OF | UNIVERSITY OF MEDICINE CALGARY

\section{CONTACT}

Kevin V. Carlson, MD, FRCSC, DABU (Fellowship Director) email: kevin.carlson@albertahealthservices.ca

\section{ANNOUNCEMENT}

\section{Fellowship In Reconstructive and Functional Urology}

\section{Offered by vesia [ALBERTA BLADDER CENTRE] and the Southern Alberta Institute of Urology, Section of Urology, Department of Surgery, University of Calgary \\ Available to qualified applicants beginning July 1, 2015}

\section{DESCRIPTION}

This is a one-year clinical fellowship. Upon completion, the fellow will be proficient in assessing and managing simple and complex presentations of incontinence, voiding dysfunction (neurogenic and non-neurogenic), pelvic organ prolapse, male stricture disease, female urologic conditions, and pelvic pain. Time will be spent in the clinic, urodynamics lab, cystoscopy suite and operating room. Technical skills will include performing and interpreting urodynamic studies (video and non-video), botulinum toxin injection, implantation of prosthetics, pelvic organ prolapse repairs (vaginal and laparoscopic approaches, including hysterectomy), other vaginal surgery (fistula repair, diverticulum excision, removal of vaginal mesh), urethroplasty, numerous surgical approaches to incontinence, cystectomy, augmentation cystoplasty, continent and incontinent urinary diversion, and ureteric reimplantation. Sacral nerve stimulation may also be available to the interested candidate. Completion and publication of clinical research projects is expected. 